\title{
Formation and Migration of Oxygen Vacancies in SrCoO and their effect on Oxygen Evolution Reactions
}

\author{
Hassan A. Tahini, Xin Tan, Udo Schwingenschlögl, and Sean C. Smith \\ ACS Catal., Just Accepted Manuscript • DOI: 10.1021/acscatal.6b00937 • Publication Date (Web): 18 Jul 2016
}

Downloaded from http://pubs.acs.org on July 24, 2016

\section{Just Accepted}

"Just Accepted" manuscripts have been peer-reviewed and accepted for publication. They are posted online prior to technical editing, formatting for publication and author proofing. The American Chemical Society provides "Just Accepted" as a free service to the research community to expedite the dissemination of scientific material as soon as possible after acceptance. "Just Accepted" manuscripts appear in full in PDF format accompanied by an HTML abstract. "Just Accepted" manuscripts have been fully peer reviewed, but should not be considered the official version of record. They are accessible to all readers and citable by the Digital Object Identifier (DOI®). "Just Accepted" is an optional service offered to authors. Therefore, the "Just Accepted" Web site may not include all articles that will be published in the journal. After a manuscript is technically edited and formatted, it will be removed from the "Just Accepted" Web site and published as an ASAP article. Note that technical editing may introduce minor changes to the manuscript text and/or graphics which could affect content, and all legal disclaimers and ethical guidelines that apply to the journal pertain. ACS cannot be held responsible for errors or consequences arising from the use of information contained in these "Just Accepted" manuscripts. 


\title{
FORMATION AND MIGRATION OF OXYGEN
} VACANCIES IN $\mathrm{SrCoO}_{3}$ AND THEIR EFFECT

\section{ON OXYGEN EVOLUTION REACTIONS}

\author{
Hassan A. Tahini ${ }^{1}$ Xin Tan ${ }^{l}$, Udo Schwingenschlögl ${ }^{2}$ and Sean C. Smith ${ }^{l^{*}}$ \\ ${ }^{1}$ Integrated Materials Design Centre (IMDC), School of Chemical Engineering, UNSW Australia, \\ Sydney, NSW 2052, Australia \\ ${ }^{2}$ Physical Science \& Engineering Division, KAUST, Thuwal 23955-6900, Kingdom of Saudi Arabia
}




\section{ABSTRACT}

Perovskite $\mathrm{SrCoO}_{3}$ is a potentially useful material for promoting the electrocatalytic oxygen evolution reaction, with high activities predicted theoretically and observed experimentally for closely related doped perovskite materials. However, complete stoichiometric oxidation is very difficult to realize experimentally - in almost all cases there are significant fractions of oxygen vacancies present. Here, using first principles calculations we study oxygen vacancies in perovskite $\mathrm{SrCoO}_{3}$ from thermodynamic, electronic and kinetic points of view. We find that an oxygen vacancy donates two electrons to neighboring Co sites in the form of localized charge. The formation energy of a single vacancy is very low and estimated to be $1.26 \mathrm{eV}$ in the dilute limit. We find that a vacancy is quite mobile with a migration energy of $\sim 0.5 \mathrm{eV}$. Moreover, we predict that oxygen vacancies exhibit a tendency towards clustering which is in accordance with the material's ability to form a variety of oxygen-deficient structures. These vacancies have a profound effect on the material's ability to facilitate OER, increasing the overpotential from $\sim 0.3 \mathrm{~V}$ for the perfect material to $\sim 0.7$ for defective surfaces. A moderate compressive biaxial strain $(2 \%)$ is predicted here to increase the surface oxygen vacancy formation energy by ca. $30 \%$, thus reducing the concentration of surface vacancies and thereby preserving the OER activity of the material.

KEYWORDS: perovskite, electrocatalyst, oxygen evolution reaction, DFT, vacancies, strain

\section{Introduction}

The search for materials with high efficiency to drive oxygen evolution reactions for electrochemical water splitting is intensely pursued for its obvious environmental benefits. ${ }^{1-3}$ The overall reaction is given by $2 \mathrm{H}_{2} \mathrm{O} \rightarrow \mathrm{O}_{2}+4 \mathrm{H}^{+}+4 \mathrm{e}^{-}$and comprises a total four proton-electron transfer steps. ${ }^{4}$ To avoid any one step acting as a bottleneck for the overall reaction, each step might ideally require $1.23 \mathrm{~V}$ in order to 
minimally match the thermodynamics of the overall process. This ideal is extremely difficult to achieve, however, and under operating conditions an applied overpotential is necessary for the reaction to proceed. To lower this overpotential, noble metals are used such as platinum and its alloys, which severely restricts the prospects of using these technologies on a commercial scale. ${ }^{2}$

Several alternatives to noble metals and their oxides have been put forward to catalyze OER. ${ }^{5,6}$ The perovskite family is a versatile family of compounds with a chemical formula $\mathrm{ABO}_{3}$. The $\mathrm{A}$ and $\mathrm{B}$ sites typically consist of alkali or alkali earth metals and transition metals, respectively, and each compound can host a range of atoms on the A or B sites acting as dopants which enriches its functional flexibility. Earlier work ${ }^{4,5,7}$ has established linear relationships between the binding energies of the various intermediates involved in the reaction, which imposes a restriction on the ability to lower the overpotential with a predicted lower bound of $0.2 \sim 0.4 \mathrm{~V}$ for oxides. ${ }^{5}$ Previous theoretical works have shown that in the family of the perovskites, $\mathrm{SrCoO}_{3}$ has theoretically the lowest overpotential of $\sim 0.25$ $\mathrm{V} .{ }^{5,8}$ However, this has been hard to demonstrate experimentally due to the fact that this material does not easily form in the ideal $\mathrm{SrCoO}_{3} \mathrm{pm} \overline{3} \mathrm{~m}$ structure but rather as an incompletely oxidized $\mathrm{SrCoO}_{3-\delta}$, where $\delta=0.5$ is the most common phase, adopting an orthorhombic crystal structure. ${ }^{9}$ Suntivich et al. ${ }^{10}$ demonstrated that $\mathrm{Ba}_{0.5} \mathrm{Sr}_{0.5} \mathrm{Co}_{0.8} \mathrm{Fe}_{0.2} \mathrm{O}_{3-\delta}$ (BSCF) can outperform the best know catalysts $\mathrm{RuO}_{2}$ or $\mathrm{IrO}_{2}$, which was explained using a descriptor based on the filling of the $e_{g}$ orbitals. However, BSCF undergoes a transformation at relatively low temperatures into new phases that do not exhibit the same performance as the parent compound. ${ }^{11}$ Recently, it was demonstrated that $\mathrm{SrCoO}_{3}$ could be stabilized in a cubic crystal form by adding scandium $(\mathrm{Sc})$ and niobium $(\mathrm{Nb})$ substitution to the B site. ${ }^{12}$ These additions where shown to enhance the electrocatalytic activity of $\mathrm{SrCoO}_{3}$ by about 6 times over BSCF at an overpotential of $0.35 \mathrm{~V}$.

In this work, we study the oxygen vacancy formation and clustering in bulk $\mathrm{SrCoO}_{3}$ as well as defective surfaces in an attempt to understand the effects of vacancies on OER. We show that vacancies in the vicinity of surface Co sites can lower their catalytic activity. To mitigate the formation of 
detrimental vacancies we propose the application of biaxial strain which suppresses $V_{O}$ formation while preserving the OER performance.

\section{Methodology}

Our calculations are based on VASP's implementation ${ }^{13}$ of density functional theory with the projector augmented waves method ${ }^{14}$ to treat core and valence electrons. The generalized gradient approximation with the PBEsol ${ }^{15}$ parameterization was used to describe the exchange-correlation, along with the RPBE ${ }^{16}$ functional which gives a better description of adsorption energies and was used previously in similar studies. ${ }^{4,5,7}$ The RPBE functional also reproduces $\mathrm{O}_{2}$ bond lengths $(1.23 \AA)$ and $\mathrm{H}_{2} \mathrm{O}$ bond lengths and angles $\left(\mathrm{H}-\mathrm{O}=0.97 \AA, \angle \mathrm{H}-\mathrm{O}-\mathrm{H}=103.6^{\circ}\right)$ that are in agreement with previous theoretical calculations performed at a different level of theory. ${ }^{17}$ Plane waves with kinetic energy cutoff of $500 \mathrm{eV}$ were used to expand wavefunctions. Energies were converged with a $1 \times 10^{-5} \mathrm{eV}$ tolerance and forces were optimized until they change by no more than $1 \times 10^{-2} \mathrm{eV} / \AA$. Adsorbed species were modelled using a 6-layer $2 \times 2 \mathrm{slab}$ with a vacuum separation of about $15 \AA$ (selected $4 \times 4$ surfaces where included for comparison in the supporting information). Dipole corrections were included in this study. ${ }^{18}$

\section{Results and Discussions}

Defect free system- We begin by looking at the structural and electronic properties of the defect free $\mathrm{SrCoO}_{3}$. $\mathrm{SrCoO}_{3}$ with a nearly full stoichiometry was found to exhibit metallic behavior along with ferromagnetic ordering. ${ }^{19}$ The formal oxidation state of the $\mathrm{B}$ sites in an $\mathrm{ABO}_{3}$ compound is generally determined by the oxidation state of the A site, which in this case leaves Co with a $4+$ charge, which allows for the possibility of several spin states due to the presence of unpaired electrons. ${ }^{20}$ Magnetic moments ranging from $1.5 \mu_{\mathrm{B}}$ to $3.0 \mu_{\mathrm{B}}$ have been predicted. ${ }^{20}$ In our model the calculated magnetic moment was found to be $1.8 \mu_{\mathrm{B}}$. This low magnetic moment qualitatively agrees with the intermediate 
spin (IS) point of view, ${ }^{21}$ but still it is underestimated due to the GGA functional used when compared with that obtained with hybrid functional for instance. ${ }^{20}$

Experimentally, $\mathrm{SrCoO}_{3-\delta}$ exists in the cubic phase for low oxygen deficiencies. Taguchi et al. ${ }^{19}$ determined the lattice parameter to be $3.836 \AA$. This value did not deviate from the those obtained by more modern and recent experiments. ${ }^{22,23}$ By fully relaxing the unit cell we obtained a cubic structure with a lattice parameter of $3.775 \AA$ with PBEsol. On the other hand, PBE and RPBE predict a lattice parameter of $3.838 \AA$ and $3.833 \AA$, respectively, which is in agreement with previous theoretical calcualtions. ${ }^{20}$ In this work we will employ the PBEsol functional to study the oxygen vacancies and their diffusion. These bulk results will be compared to those obtained using RPBE which will later be used to study the adsorption of OER intermediates.

Oxygen vacancies- We simulate the effect of oxygen vacancies in $\mathrm{SrCoO}_{3}$ using a $2 \times 2 \times 2$ supercell by removing one $\mathrm{O}$ atom from its normal site. This creates an $\mathrm{O}-\mathrm{Co}-\mathrm{V}_{\mathrm{O}}$ oriented along the $\mathrm{z}$-axis to simplify orbital analysis (by aligning the local d-orbitals with the global projection axis used by the DFT code). The removal of one neutral $\mathrm{O}$ atom leads to two electrons being left behind in the lattice. Generally, these electrons could end up localized on neighboring metal sites or delocalized through the lattice. 
(a)
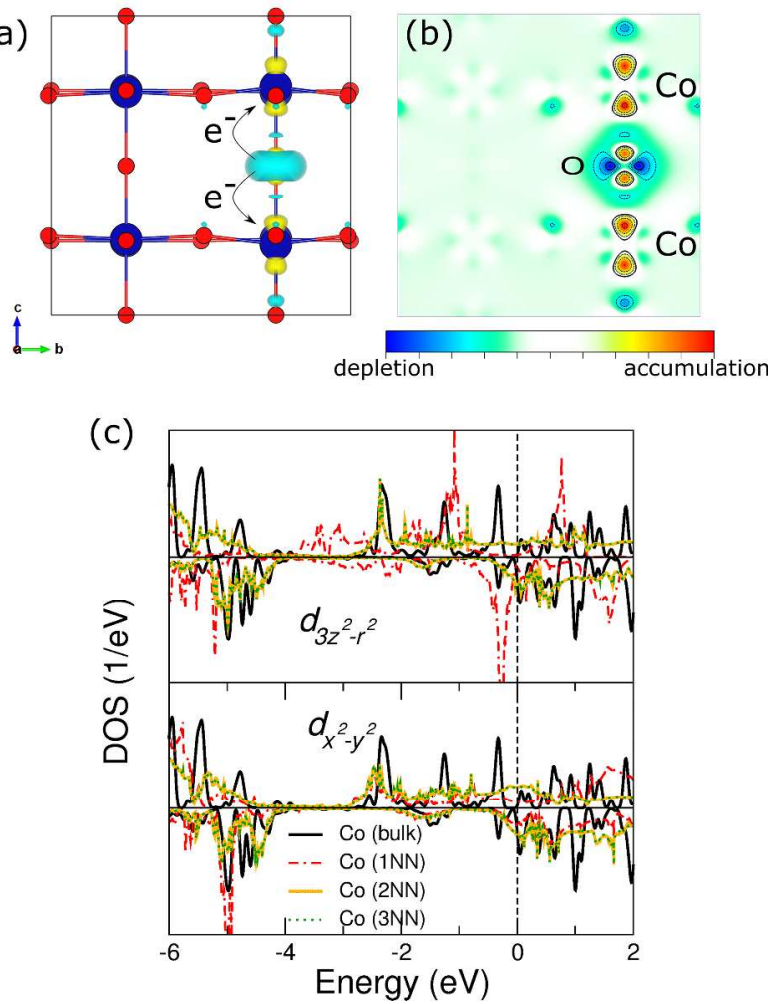

Figure 1. (a),(b) Differential charge density upon the creation of $\mathrm{V}_{\mathrm{O}}$ and a slice along the (100) plane. (c) The $d_{3 z 2-\mathrm{r} 2}$ and $d_{\mathrm{x} 2-\mathrm{y} 2}$ contributions of the Co ions in a pristine bulk system and the Co ions surrounding $\mathrm{V}_{\mathrm{O}}$. Figures were rendered using VESTA. ${ }^{24}$

The charge density redistribution caused by the creation of the vacancy is given with respect to the charge density of the vacancy containing supercell $\left[\rho\left(V_{O}\right)\right]$, the bulk system $[\rho($ bulk $)]$ and a single $\mathrm{O}$ atom added at the vacancy site $[\rho(\mathrm{O})]$ and is given by $\Delta \rho=\rho\left(\mathrm{V}_{\mathrm{O}}\right)+\rho(\mathrm{O})-\rho$ (bulk). The resulting differential charge density is shown in Fig.1(a),(b). Here, it is clear that $\mathrm{V}_{\mathrm{O}}$ donates charge to the two neighboring Co ions. The shape of the accumulated charge suggest that the donated electrons go into $d_{3 z^{2}-r^{2}}$ orbitals centered on the Co sites, while the depleted charge regions on the $\mathrm{O}$ ion suggests that this charge originates from the $p_{y}$ orbitals. Further evidence for charge accumulating in the $d_{3 z^{2}-r^{2}}$ orbitals is given by the DOS plots shown in Fig.1(c). The local DOS (LDOS) for the Co ions closest to the vacancy show 
a peak right below the Fermi level, for which the integrated profile between -0.5 and the Fermi level gives $1 e^{-}$

The oxygen formation energy was calculated according to:

$$
E_{f}\left(V_{\mathrm{O}}\right)=E\left(V_{\mathrm{O}}\right)-E(\text { perf })+\frac{1}{2}\left[\Delta h+E\left(\mathrm{O}_{2}\right)\right]
$$

Where $E\left(V_{\mathrm{O}}\right), E$ (perf) and $E\left(\mathrm{O}_{2}\right)$ are the total energies of the defect containing supercell, perfect supercell and the energy of an $\mathrm{O}_{2}$ molecule. $\Delta h$ is a correction term added to account for the well-known $\mathrm{O}_{2}$ overbinding in DFT calculations. This value is obtained by fitting the heats of formation of a range of oxides and determining the offset from the well characterized experimentally determined heats of formation. ${ }^{25,26}$ Using this approach we find the correction with PBEsol to be $0.9 \mathrm{eV} / \mathrm{O}_{2}\left(1.7 \mathrm{eV} / \mathrm{O}_{2}\right.$ using RPBE and $1.4 \mathrm{eV} / \mathrm{O}_{2}$ using PBE which is consistent with previous theoretical calculations ${ }^{25-27}$ ). The vacancy formation energy was computed for a number of supercell sizes which then allows the extrapolation to a dilute limit. These are, the $2 \times 2 \times 2,3 \times 3 \times 3$ and $4 \times 4 \times 4$ cubic cells and the $a \sqrt{2} \times a \sqrt{2}$, $2 a \sqrt{2} \times 2 a \sqrt{2}$ and the $3 a \sqrt{2} \times 3 a \sqrt{2}$ rhombohedral cells. The obtained formation energies are then fitted to $E_{f}(L)=E_{\infty}+a L^{-1}+b L^{-3}$ to account for electrostatic and elastic interactions. ${ }^{28,29}$

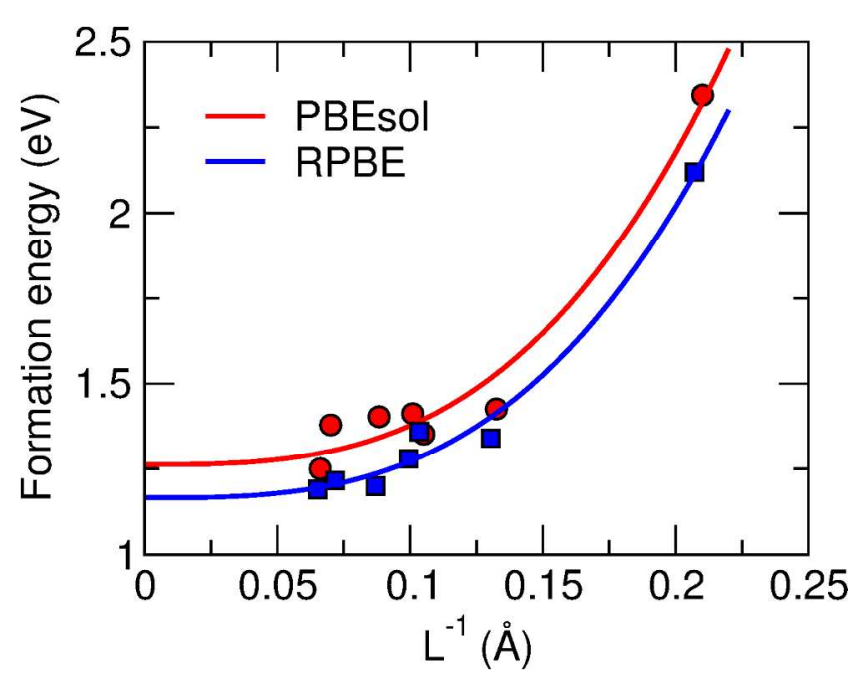


Figure 2. The oxygen vacancy formation energy calculated as a function of cell size using cubic and rhombohedral cells. The data points are fit into a polynomial of the form $E_{f}(L)=E_{\infty}+a L^{-1}+b L^{-3}$ and is represented by the solid line.

The extrapolated value to infinity (Fig. 2) puts the formation energy of a single vacancy at $1.26 \mathrm{eV}$ in the oxygen rich regime (1.17 eV with RPBE). This is much lower than other typical perovskites. For instance in $\mathrm{LaNiO}_{3}$ this value was determined to be $2.28 \mathrm{eV}^{29}$ and $2.7 \mathrm{eV}$ for $\mathrm{La}_{0.75} \mathrm{Sr}_{0.25} \mathrm{MnO}_{3}{ }^{30} \mathrm{On}$ the other hand, the value is more comparable to BSCF which was found to be $1.34 \sim 1.40 \mathrm{eV}^{11}$ (depending on local environment). This low formation energy suggests a high concentration of $\mathrm{V}_{\mathrm{O}}$ even under O-rich conditions. $\mathrm{V}_{\mathrm{O}}$ concentration will further increase at lower partial pressures of oxygen signalling a preference to form non-stoichiometric $\mathrm{SrCoO}_{3-\delta}$.

Oxygen divacancy interactions- To shed some light on the interaction of oxygen vacancies in $\mathrm{SrCoO}_{3}$, we used a $2 \times 2 \times 2$ supercell consisting of eight unit cells. We considered two $\mathrm{V}_{\mathrm{O}}$ at a time. The atomic positions were relaxed without taking into account the distortions and deformations to the supercell volume and shape that might be caused by the presence of the vacancies, which we will consider separately. The chosen supercell offers seven unique arrangements of the $V_{O}$ (see Fig. 3). ${ }^{29,31}$ To assess the extent of the vacancy interactions, we calculated the binding according to:

$$
E_{b}\left(2 V_{O}\right)=E\left(2 V_{O}\right)-2 E\left(V_{O}\right)+E(\text { perf })
$$

Where $E\left(2 V_{O}\right)$ is the total energy of the system containing a divacancy. Here, a negative binding energy corresponds to a stable cluster relative to two $V_{O}$ that are infinitely separated. 

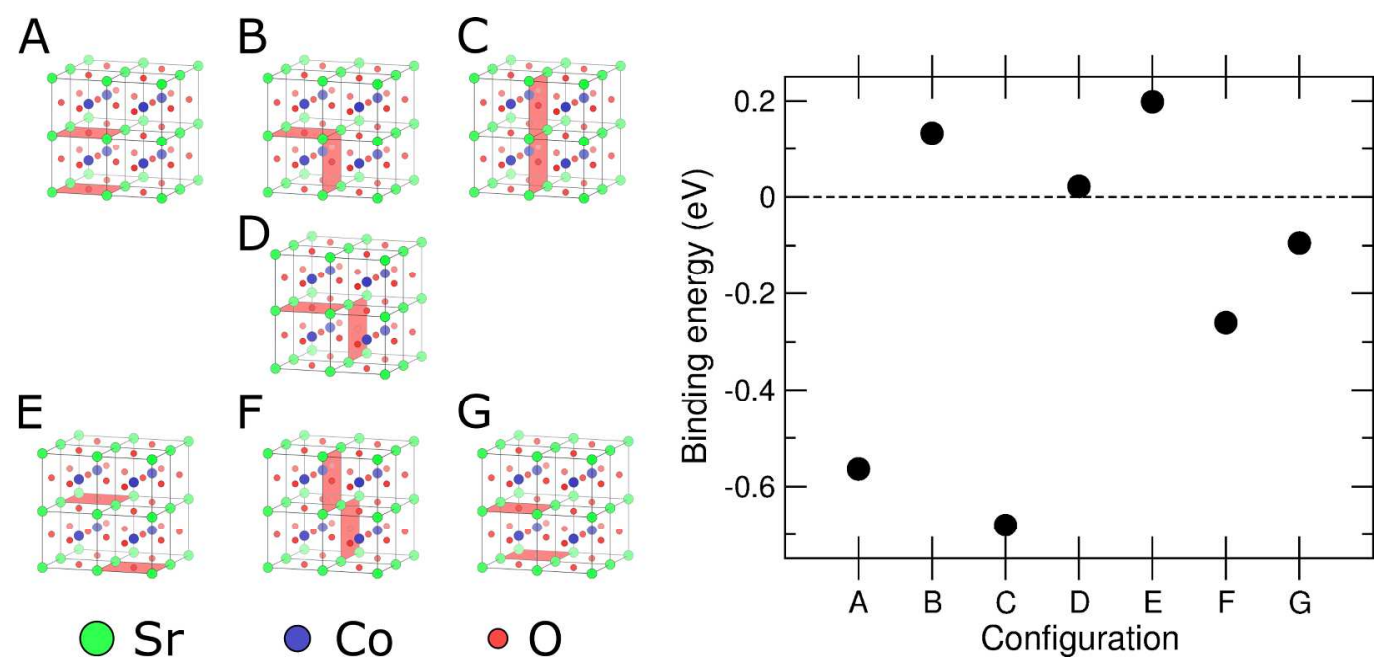

Figure 3. The left panel depicts the divacancy configurations labelled A-G. Shown on the right are the binding energies corresponding to each configuration. The shaded planes represent the positions of the oxygen vacancies.

Fig. 3 shows that several configurations are stable, in particular configuration $\mathrm{C}$ depicting two vacancies in the same plane in a basal site of the $\mathrm{CoO}_{6}$ octahedron. The next most stable cluster is configuration A which represents an apical divacancy (the two vacancies are separated by a Co ion). Previous studies have showed that this is the stable configuration in $\mathrm{SrTiO}_{3}{ }^{31}$ and $\mathrm{LaNiO}_{3}{ }^{29}$. The tendency of $\mathrm{V}_{\mathrm{O}}$ to exist in bound clusters is in agreement with the established observation that $\mathrm{SrCoO}_{3}$ can easily form oxygen deficient structures. Moreover, the preference for certain orientations such as the basal or apical divacancy configurations could explain the transition to brownmillerite phase which is a vacancy ordered structure..$^{9,32,33}$

Oxygen migration in $\mathrm{SrCoO}_{3}$ - In general, theoretical calculations have revealed that for a number of perovskites $\mathrm{O}$ ions migrate from one vacant site to another via a curved migration pathway. ${ }^{34,35}$ We have confirmed that this is also the case for $\mathrm{SrCoO}_{3}$ (see Fig. 4(b)). Our calculations were performed in a 40, 135 and 320 atom supercells and the migration energies were found to be $0.78 \mathrm{eV}, 0.5 \mathrm{eV}$ and $0.56 \mathrm{eV}$, respectively. These values are still lower than the oxygen migration in $\operatorname{SrCoO}_{2.5}(\delta=0.5)$ in the 
brownmillerite phase which has a different crystal structure but nevertheless a similar oxygen environment for the octahedral layers in which the calculated barrier is $0.82 \mathrm{eV} .{ }^{36}$ However, our values are similar to those in $\mathrm{Ba}_{0.5} \mathrm{Sr}_{0.5} \mathrm{CoO}_{3}(0.40 \mathrm{eV}) .{ }^{37}$

(a)
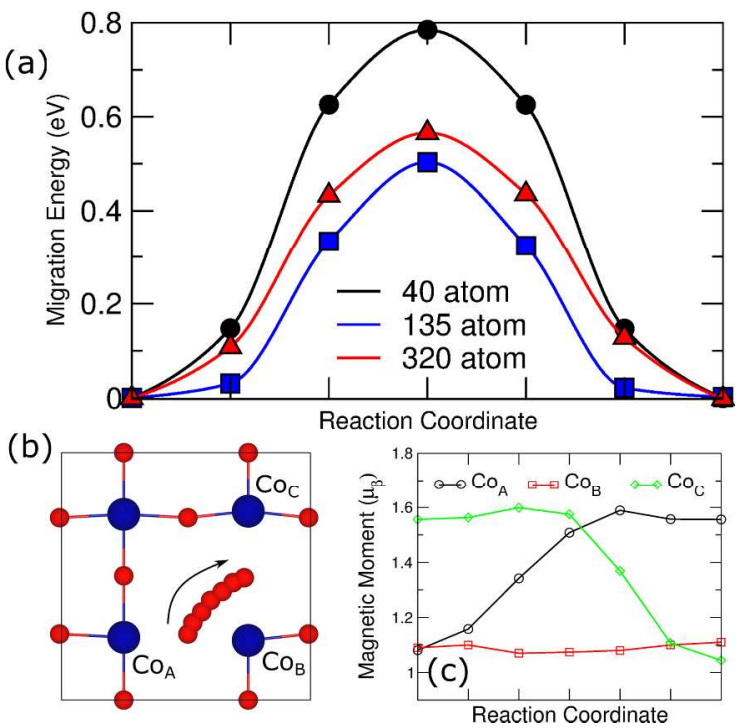

Figure 4. (a) The migration energy barriers for $V_{O}$ in a 40 atom, 135 atom and 320 atom supercells. (b) The curved migration pathway followed by the diffusing $\mathrm{O}$ atom. (c) The magnetic moments of the nearest neighbor Co ions involved in the migration process.

The Co ions in the proximity of the migrating $\mathrm{O}$ ion are shown in Fig. $4(\mathrm{~b}) . \mathrm{Co}_{\mathrm{B}}$ remains coordinated to the migrating $\mathrm{O}$ ion (and the vacant site) through its transition from the initial to the final state. As such its magnetic moment remains low throughout. On the other hand, $\mathrm{Co}_{\mathrm{A}}$ and $\mathrm{Co}_{\mathrm{C}}$ are coordinated to the vacancy in the final and initial states respectively, which leads to a nearly symmetric change in $\mu_{\mathrm{Co}_{\mathrm{A}}}$ and $\mu_{\mathrm{Co}_{\mathrm{C}}}$ between the initial and final states. This change is not as abrupt as in other perovksites ${ }^{34,38}$ which is an indication of the covalent character of Co-O bonding.

OER with oxygen vacancies- While it has been inferred from theoretical calculations ${ }^{5,8}$ and experiments ${ }^{39,40}$ that $\mathrm{SrCoO}_{3}$ is one of the best materials for OER, a clear experimental verification remains difficult. Experimentally, it remains challenging to grow fully oxidized thin films of $\mathrm{SrCoO}_{3} \cdot{ }^{12,32}$ 
Zhou et al. ${ }^{12}$ also noticed a reduced OER activity when phases other than the cubic phase were present. Therefore, it is important to understand how oxygen vacancies alter the surface properties of $\mathrm{SrCoO}_{3}$ and how they interact with OER intermediates. We consider an oxygen vacancy on the surface of the (001) plane and another subsurface vacant $\mathrm{O}$ site to see how this could alter the activity of the Co sites. For this part of the work we used the RPBE functional which is better for handling adsorption energies. ${ }^{4,5,26}$ The workfunction of the bare (001) surface is $6.00 \mathrm{eV}(6.35 \mathrm{eV}$ with PBEsol). This corresponds to $1.56 \mathrm{~V}$ vs SHE which indicates a strong oxidizing potential. When introducing an oxygen vacancy the workfunctions become 5.85 and $5.97 \mathrm{eV}$ for the surface and subsurface sites, respectively.

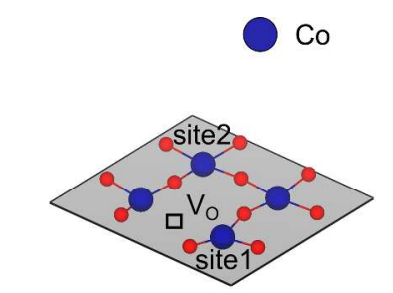

- $\mathrm{O} \quad \square V_{0}$

(a)

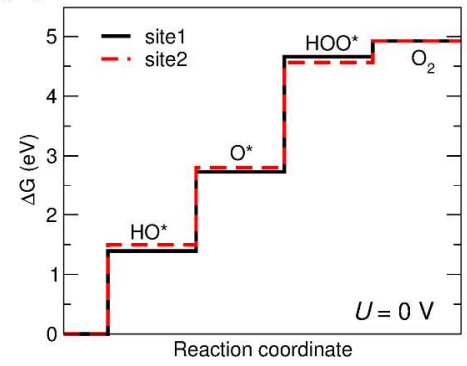

(b)
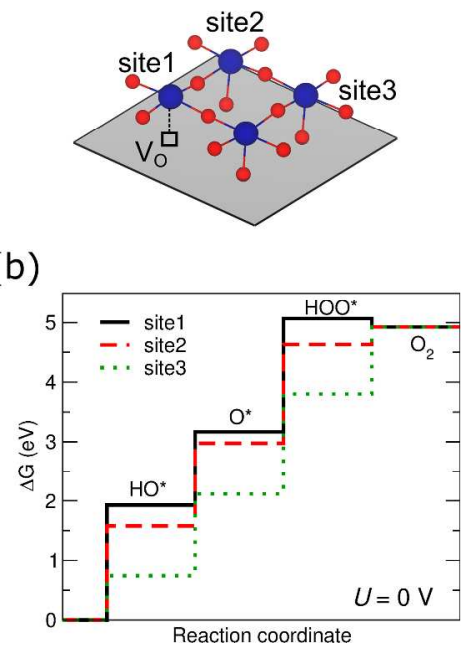

Figure 5. The free energy for each reaction step on different Co sites for (a) surface and (b) subsurface $\mathrm{V}_{\mathrm{O}}$. The surface and subsurface oxygen vacancies are shown in the left and right panels, respectively. The distinct active sites for the $2 \times 2$ surface in the presence of the vacancy are as indicated.

Fig. 5 shows the free energy profiles of the OER steps. When the electrode potential is $0 \mathrm{~V}$ all reaction steps are uphill. For surface $V_{O}$, the Co sites shown in Fig. 5(a) exhibit different activities. While on both sites the step $\mathrm{O}^{*} \rightarrow \mathrm{HOO}^{*}$ is rate determining, we find that the energy required for this step increases at the site in the immediate vicinity of $\mathrm{V}_{\mathrm{O}}$, leading to an overpotential of $0.7 \mathrm{~V}$ and $0.52 \mathrm{~V}$ for site 1 and site2, respectively. This is $0.2 \sim 0.4 \mathrm{~V}$ higher than the perfect $\mathrm{SrCoO}_{3}$ surface. Similarly, subsurface $\mathrm{V}_{\mathrm{O}}$ 
leads to a reduction in the activity of the Co site immediately above it (site1) for which the overpotential is $0.7 \mathrm{~V}$, and rate determining step is the dissociation of a water molecule to $\mathrm{HO}^{*}$ which is only slightly higher than the formation of $\mathrm{HOO}^{*}$. Moving away from the vacant site, the activity improves with overpotentials calculated to $\sim 0.45 \mathrm{~V}$.

The presence of vacancies causes a local distortion in the Co-O-Co bonds. For instance, for surface $\mathrm{V}_{\mathrm{O}}$ the Co-O-Co angle was found to be $164^{\circ}$. Additionally, as we showed earlier, $\mathrm{V}_{\mathrm{O}}$ donates its electrons to it neighboring Co ions, thus reducing them. These reduced surface Co sites along with the buckled Co-OCo bonds leads to a decrease in the overlap between the Co $3 d$ and O $2 p$ orbitals leading to a reduction in the electron transfer between the surface and the OER intermediates. ${ }^{40}$ This deterioration in activity due to the presence of oxygen vacancies have been recently suggested by Cheng et al. ${ }^{40}$

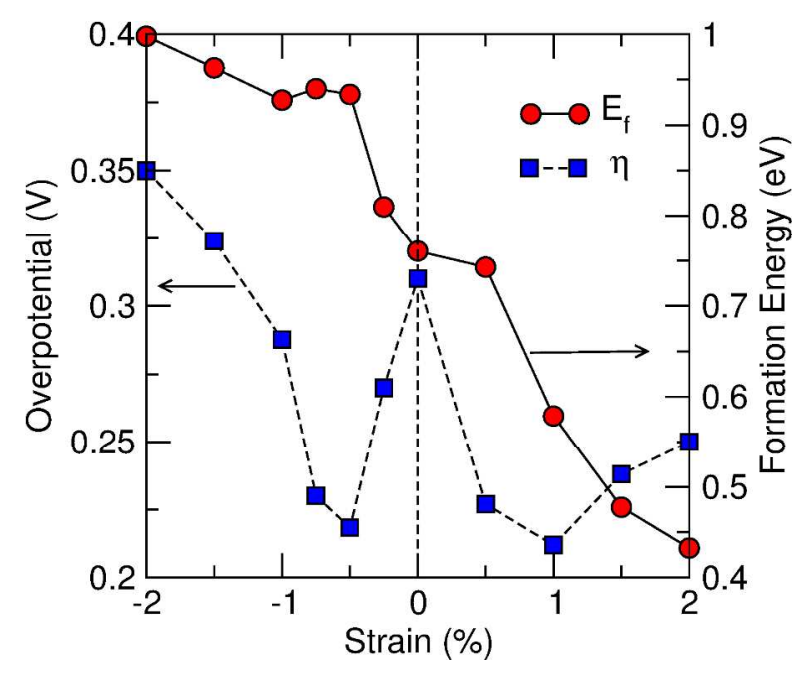

Figure 6. Surface VO formation energy and overpotential for OER plotted against strain. The formation energy increases with biaxial compressive strain while the overpotential remains close to the unstrained case.

Finding means to suppress the formation of these surface vacancies would be highly valuable. To this end, strain engineering has been successfully applied to tune electronic and thermodynamic properties of 
solids. In this case we find that the application of moderate biaxial compressive strain leads to an increase in surface $V_{O}$ formation energy (Fig. 6). Application of hydrostatic or compressive strain on perovskites straightens the B-O-B bonds. ${ }^{41,42}$ Compression which favors Co-O-Co angles closer to $180^{\circ}$ competes with tendency of a vacancy to distort the lattice hence disfavoring its formation which increases to $1 \mathrm{eV}$ at a $-2 \%$ strain compared to $0.75 \mathrm{eV}$ in the unstrained case. On the other hand, the OER activity in the moderately strained structures remains close to the ideal case with a predicted overpotential of $0.31 \mathrm{~V}$. A similar approach was used recently to control oxygen vacancies in brownmillerite $\mathrm{SrCoO}_{\mathrm{x}}{ }^{43}$ While this increase in the formation energy does not completely eliminate surface oxygenated species, it should provide a way forward to achieve a higher capacity of the catalytic power of $\mathrm{SrCoO}_{3}$.

\section{Conclusions}

In this work we have studied the electronic, thermodynamics and kinetic aspects of the oxygen vacancies in $\mathrm{SrCoO}_{3}$. We find that creation of an oxygen vacancy effectively donates two electrons to neighboring Co sites, thereby reducing them. These vacancies have low formation energies which are calculated to be $1.17-1.26 \mathrm{eV}$ in the dilute limit. The migration barrier for a diffusing oxygen vacancy was found to be $\sim 0.5 \mathrm{eV}$, which suggests that this defect is quite mobile relative to other typical perovskites. ${ }^{29,31,34}$ In accord with the high oxygen deficiencies reported in experiments, we find that in the context of divacancies this material has a tendency to form clustered $\mathrm{V}_{\mathrm{O}}$ which are aligned in a basal or apical configuration. Finally, we have investigated the effect of surface vacancies on the OER activity and we found oxygen vacancies are detrimental to the materials performance, in line with previous experiments. ${ }^{40}$ Applying biaxial compressive strain increases the energetic cost of forming these defects and does not negatively impact catalytic activity compared with the unstrained pristine perovskite (001) surface, suggesting a possible route to minimizing surface vacancies and thereby optimizing the catalytic performance. 


\title{
ASSOCIATED CONTENT
}

Supporting Information. Details of calculations and models used. This material is available free of charge via the Internet at http://pubs.acs.org.

\author{
AUTHOR INFORMATION \\ Corresponding Author \\ * Sean C. Smith: sean.smith@unsw.edu.au
}

\section{ACKNOWLEDGEMENT}

This research was undertaken with the assistance of UNSW Australia SPF01 funding (SCS). We acknowledge generous allocations of supercomputing time at the Pawsey Supercomputing Centre via the Australian National Computational Merit Allocation Scheme (NCMAS project fr2) and the Energy and Resources Merit Allocation Scheme of the Pawsey Supercomputing Centre (project pawsey0111). Additional computational resources were provided by KAUST on the Shaheen II supercomputer.

\section{REFERENCES}

(1) Walter, M. G.; Warren, E. L.; McKone, J. R.; Boettcher, S. W.; Mi, Q.; Santori, E. a.; Lewis, N. S. Chem. Rev. 2010, 110, 6446-6473.

(2) Katsounaros, I.; Cherevko, S.; Zeradjanin, A. R.; Mayrhofer, K. J. J. Angew. Chemie Int. Ed. 2014, $53,102-121$.

(3) Li, Y.-F.; Liu, Z.-P. Wiley Interdiscip. Rev. Comput. Mol. Sci. 2016, 6, 47-64. 
(4) Nørskov, J. K.; Rossmeisl, J.; Logadottir, A.; Lindqvist, L.; Kitchin, J. R.; Bligaard, T.; Jónsson, H. J. Phys. Chem. B 2004, 108, 17886-17892.

(5) Man, I. C.; Su, H.-Y.; Calle-Vallejo, F.; Hansen, H. a.; Martínez, J. I.; Inoglu, N. G.; Kitchin, J.; Jaramillo, T. F.; Nørskov, J. K.; Rossmeis1, J. ChemCatChem 2011, 3, 1159-1165.

(6) Zhang, J.; Zhao, Z.; Xia, Z.; Dai, L. Nat. Nanotechnol. 2015, 10, 444-452.

(7) Rossmeisl, J.; Logadottir, A.; Nørskov, J. K. Chem. Phys. 2005, 319, 178-184.

(8) Calle-Vallejo, F.; Díaz-Morales, O. A.; Kolb, M. J.; Koper, M. T. M. ACS Catal. 2015, 5, 869873.

(9) Muñoz, A.; de la Calle, C.; Alonso, J. A.; Botta, P. M.; Pardo, V.; Baldomir, D.; Rivas, J. Phys. Rev. B 2008, 78, 54404.

(10) Suntivich, J.; May, K. J.; Gasteiger, H. A.; Goodenough, J. B.; Shao-Horn, Y. Science 2011, 334, $1383-1385$.

(11) Kuklja, M. M.; Mastrikov, Y. A.; Jansang, B.; Kotomin, E. a. Solid State Ionics 2013, 230, 21-26.

(12) Zhou, W.; Zhao, M.; Liang, F.; Smith, S. C.; Zhu, Z. Mater. Horiz. 2015, 2, 495-501.

(13) Kresse, G.; Furthmüller, J. Phys. Rev. B 1996, 54, 11169-11186.

(14) Kresse, G.; Joubert, D. Phys. Rev. B 1999, 59, 1758-1775.

(15) Perdew, J. P.; Ruzsinszky, A.; Csonka, G. I.; Vydrov, O. A.; Scuseria, G. E.; Constantin, L. A.; Zhou, X.; Burke, K. Phys. Rev. Lett. 2008, 100, 136406.

(16) Hammer, B.; Hansen, L. B.; Nørskov, J. K. Phys. Rev. B 1999, 59, 7413-7421.

(17) Xu, X.; Goddard, W. A. J. Phys. Chem. A 2004, 108, 2305-2313. 
(18) Neugebauer, J.; Scheffler, M. Phys. Rev. B 1992, 46, 16067-16080.

(19) Taguchi, H.; Shimada, M.; Koizumi, M. J. Solid State Chem. 1979, 29, 221-225.

(20) Hoffmann, M.; Borisov, V. S.; Ostanin, S.; Mertig, I.; Hergert, W.; Ernst, A. Phys. Rev. B 2015, $92,94427$.

(21) Mastrikov, Y. a; Kuklja, M. M.; Kotomin, E. a; Maier, J. Energy Environ. Sci. 2010, 3, 1544.

(22) Bezdicka, P.; Wattiaux, A.; Grenier, J. C.; Pouchard, M.; Hagenmuller, P. Zeitschrift für Anorg. und Allg. Chemie 1993, 619, 7-12.

(23) Long, Y.; Kaneko, Y.; Ishiwata, S.; Taguchi, Y.; Tokura, Y. J. Phys. Condens. Matter 2011, 23, 245601

(24) Momma, K.; Izumi, F. J. Appl. Crystallogr. 2011, 44, 1272-1276.

(25) Wang, L.; Maxisch, T.; Ceder, G. Phys. Rev. B 2006, 73, 195107.

(26) Lee, Y.-L.; Kleis, J.; Rossmeisl, J.; Morgan, D. Phys. Rev. B 2009, 80, 224101.

(27) Chen, D.; Chen, C.; Zhang, Z.; Baiyee, Z. M.; Ciucci, F.; Shao, Z. ACS Appl. Mater. Interfaces 2015, 7, 8562-8571.

(28) Castleton, C. W. M.; Mirbt, S. Phys. Rev. B 2004, 70, 195202.

(29) Malashevich, A.; Ismail-Beigi, S. Phys. Rev. B 2015, 92, 144102.

(30) Mastrikov, Y. A.; Merkle, R.; Heifets, E.; Kotomin, E. A.; Maier, J. J. Phys. Chem. C 2010, 114, $3017-3027$.

(31) Cuong, D. D.; Lee, B.; Choi, K. M.; Ahn, H.-S.; Han, S.; Lee, J. Phys. Rev. Lett. 2007, 98, 115503. 
(32) Jeen, H.; Choi, W. S.; Freeland, J. W.; Ohta, H.; Jung, C. U.; Lee, H. N. Adv. Mater. 2013, 25, 3651-3656.

(33) Jeen, H.; Bi, Z.; Choi, W. S.; Chisholm, M. F.; Bridges, C. a.; Paranthaman, M. P.; Lee, H. N. Adv. Mater. 2013, 25, 6459-6463.

(34) Ritzmann, A. M.; Muñoz-García, A. B.; Pavone, M.; Keith, J. A.; Carter, E. A. Chem. Mater. 2013, 25, 3011-3019.

(35) Jones, A.; Islam, M. S. J. Phys. Chem. C 2008, 112, 4455-4462.

(36) Mitra, C.; Meyer, T.; Lee, H. N.; Reboredo, F. A. J. Chem. Phys. 2014, 141, 84710.

(37) Kotomin, E. a.; Mastrikov, Y. a.; Kuklja, M. M.; Merkle, R.; Roytburd, a.; Maier, J. Solid State Ionics 2011, 188, 1-5.

(38) Ritzmann, A. M.; Pavone, M.; Muñoz-García, A. B.; Keith, J. A.; Carter, E. A. J. Mater. Chem. A 2014, 2, 8060-8074.

(39) Matsumoto, Y. J. Electrochem. Soc. 1980, 127, 811.

(40) Cheng, X.; Fabbri, E.; Nachtegaal, M.; Castelli, I. E.; El Kazzi, M.; Haumont, R.; Marzari, N.; Schmidt, T. J. Chem. Mater. 2015, 27, 7662-7672.

(41) Loa, I.; Adler, P.; Grzechnik, A.; Syassen, K.; Schwarz, U.; Hanfland, M.; Rozenberg, G. K.; Gorodetsky, P.; Pasternak, M. P. Phys. Rev. Lett. 2001, 87, 125501.

(42) Ross, N. L.; Zhao, J.; Angel, R. J. J. Solid State Chem. 2004, 177, 3768-3775.

(43) Petrie, J. R.; Mitra, C.; Jeen, H.; Choi, W. S.; Meyer, T. L.; Reboredo, F. A.; Freeland, J. W.; Eres, G.; Lee, H. N. Adv. Funct. Mater. 2016, 26, 1564-1570. 
Table of Contents Graphic and Synopsis

$\mathrm{SrCoO}_{3}$ is one of the best materials for oxygen evolution reaction. However, the presence of oxygen vacancies interferes with its catalytic properties leading to an increase in overpotentials from those predicted for the ideal surfaces. Compressive biaxial strain can suppress the formation of surface oxygen vacancies without a significant effect on the overpotential making this an attractive pathway to harness the material's full catalytic power.

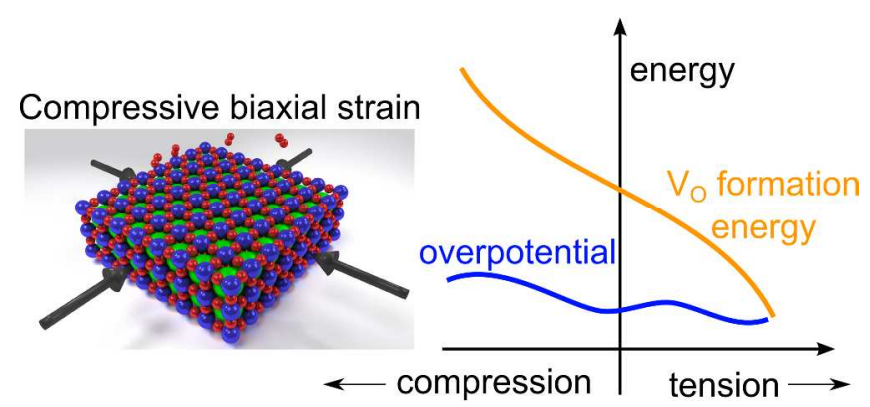

